University of Wollongong

Research Online

Australian Institute for Innovative Materials -

Papers

Australian Institute for Innovative Materials

$1-1-2013$

Critical phenomena and estimation of the spontaneous magnetization by a magnetic entropy analysis in Mn0.96 Nb0.04CoGe alloy

Jyotish C. Debnath

University Of Wollongong, jcd341@@uow.edu.au

A M. Strydom

University of Johannesburg

P Shamba

ps807@uowmail.edu.au

Jianli Wang

University of Wollongong, jianli@uow.edu.au

S X. Dou

University of Wollongong, shi@uow.edu.au

Follow this and additional works at: https://ro.uow.edu.au/aiimpapers

Part of the Engineering Commons, and the Physical Sciences and Mathematics Commons

Research Online is the open access institutional repository for the University of Wollongong. For further information contact the UOW Library: research-pubs@uow.edu.au 


\title{
Critical phenomena and estimation of the spontaneous magnetization by a magnetic entropy analysis in Mn0.96Nb0.04CoGe alloy
}

\author{
Abstract \\ Magnetic and magnetocaloric properties of the alloy Mn0.96Nb0.04CoGe have been investigated. \\ Keywords \\ $\mathrm{mn} 0,96 \mathrm{nb0}$, magnetization, 04coge, magnetic, alloy, spontaneous, estimation, phenomena, critical, \\ entropy, analysis \\ Disciplines \\ Engineering | Physical Sciences and Mathematics

\section{Publication Details} \\ Debnath, J. C., Strydom, A. M., Shamba, P., Wang, J. \& Dou, S. X. (2013). Critical phenomena and \\ estimation of the spontaneous magnetization by a magnetic entropy analysis in Mn0.96Nb0.04CoGe \\ alloy. Journal of Applied Physics, 113 (23), 233903-1-233903-5.
}




\section{AIP $\mid$ Applied Physics}

Critical phenomena and estimation of the spontaneous magnetization by a magnetic entropy analysis in Mn0.96Nb0.04CoGe alloy

J. C. Debnath, A. M. Strydom, P. Shamba, J. L. Wang, and S. X. Dou

Citation: J. Appl. Phys. 113, 233903 (2013); doi: 10.1063/1.4811342

View online: http://dx.doi.org/10.1063/1.4811342

View Table of Contents: http://jap.aip.org/resource/1/JAPIAU/v113/i23

Published by the AIP Publishing LLC.

Additional information on J. Appl. Phys.

Journal Homepage: http://jap.aip.org/

Journal Information: http://jap.aip.org/about/about_the_journal

Top downloads: http://jap.aip.org/features/most_downloaded

Information for Authors: http://jap.aip.org/authors

\section{ADVERTISEMENT}

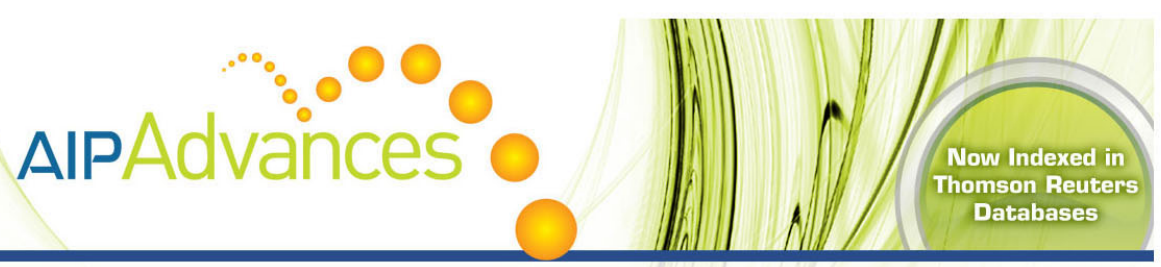

\section{Explore AIP's open access journal: Rapid publication \\ - Article-level metrics \\ - Post-publication rating and commenting}




\title{
Critical phenomena and estimation of the spontaneous magnetization by a magnetic entropy analysis in $\mathrm{Mn}_{\mathbf{0 . 9 6}} \mathrm{Nb}_{0.04}$ CoGe alloy
}

\author{
J. C. Debnath, ${ }^{1, a)}$ A. M. Strydom, ${ }^{1}$ P. Shamba, ${ }^{2}$ J. L. Wang, ${ }^{2,3}$ and S. X. Dou ${ }^{2}$ \\ ${ }^{1}$ Department of Physics, University of Johannesburg, P.O. Box 524, Auckland Park 2006, South Africa \\ ${ }^{2}$ Institute for Superconducting and Electronic Materials, University of Wollongong, Squaire Way, North \\ Wollongong, Wollongong, NSW 2500, Australia \\ ${ }^{3}$ Bragg Institute, Australian Nuclear Science and Technology Organization, Lucas Heights, NSW 2234, \\ Australia
}

(Received 22 April 2013; accepted 31 May 2013; published online 17 June 2013)

\begin{abstract}
Magnetic and magnetocaloric properties of the alloy $\mathrm{Mn}_{0.96} \mathrm{Nb}_{0.04} \mathrm{CoGe}$ have been investigated. According to the mean-field theory prediction, the relationship between $\Delta S_{M} \propto\left(H / T_{C}\right)^{2 / 3}$ has been confirmed in the temperature region near $T_{C}$ for that system. To investigate the nature of the magnetic phase transition, a detailed critical exponent study has been performed. The critical components, $\gamma, \beta$, and $\delta$ determined using the Kouvel-Fisher method, the modified Arrott plot, as well as the critical isotherm analysis agree well. Moreover, these critical exponents are confirmed by the Widom scaling law and the validity of the calculated critical exponents was also confirmed by the scaling theory. The values deduced for the critical exponents are close to the theoretical prediction of the mean-field model values, thus indicating that long range interactions dominate the critical behavior in the $\mathrm{Mn}_{0.96} \mathrm{Nb}_{0.04} \mathrm{CoGe}$ system. It is also speculated that the competition between the localized Mn-Mn magnetic interactions should be responsible for the critical behavior in this system. Moreover, an excellent agreement is found between the spontaneous magnetization determined from the entropy change $\left(-\Delta S_{M}\right.$ vs. $\left.M^{2}\right)$ and the classical extrapolation from the Arrott curves $\left(H / M\right.$ vs. $\left.M^{2}\right)$, thus confirming that the magnetic entropy change is a valid approach to estimate the spontaneous magnetization in this system. (C) 2013 AIP Publishing LLC. [http://dx.doi.org/10.1063/1.4811342]
\end{abstract}

\section{INTRODUCTION}

In recent years, magnetic materials with large magnetocaloric effect (MCE) have been extensively studied both experimentally and theoretically due to their great potential applications in magnetic refrigeration. ${ }^{1,2}$ This new cooling technology which is expected to supersede the conventional refrigeration technology based on gas-compression/expansion is of special interest because of its considerable economic benefits. ${ }^{3,4}$ Significant advances have been made in interpreting the magnetocaloric properties of materials. The Landau theory for phase transitions is applied to describe the MCE in ferromagnetic (FM) systems with magnetoelastic and magnetoelectronic couplings. ${ }^{5,6}$ Moreover, the meanfield theory has established direct relations between magnetic entropy change and magnetization. The theory of critical phenomena justifies the existence of a universal magnetocaloric behavior in materials presenting secondorder magnetic phase transitions. ${ }^{7,8}$ Thus, studies of critical exponents can supply valuable information about magnetic phase transitions.

Recently, a lot of research interest has been devoted to the $\mathrm{MnCoGe}$ material system for use as a magnetic refrigerant. ${ }^{9,10}$ This material system undergoes a second order magnetic phase transition as well as a crystallographic phase transition from the low temperature orthorhombic TiNiSi

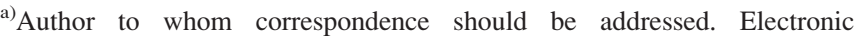
addresses: jcd341@uowmail.edu.au and jdebnath@uj.ac.za.
}

type to the high temperature hexagonal $\mathrm{Ni}_{2}$ In type structure. ${ }^{11}$ Although different properties of $\mathrm{MnCoGe}$ have been extensively investigated, more studies are desired to understand the intrinsic magnetic interactions. Recent investigations have revealed that an effective way of probing the magnetic interactions responsible for the magnetic transitions is by performing a critical exponent analysis in the vicinity of the FM-paramagnetic (PM) region. ${ }^{12,13}$ Thus, in an effort to understand the nature of the magnetic transition in $\mathrm{Mn}_{0.96} \mathrm{Nb}_{0.04} \mathrm{CoGe}$, we performed a critical exponent study by using different methods.

In this paper, we study the magnetocaloric properties and critical behaviour of the $\mathrm{Mn}_{0.96} \mathrm{Nb}_{0.04} \mathrm{CoGe}$ alloy. For the understanding of the nature of magnetic transition in $\mathrm{Mn}_{0.96} \mathrm{Nb}_{0.04} \mathrm{CoGe}$, we performed a critical exponent analysis in the vicinity of the (FM)-(PM) region, where the critical exponents $\beta, \gamma$, and $\delta$ have been obtained reliably by different analytical methods. ${ }^{12,13}$ It is found that the magnetic behavior of $\mathrm{Mn}_{0.96} \mathrm{Nb}_{0.04} \mathrm{CoGe}$ alloy is close to the theoretical prediction of the mean-field model. Apart from a slight decrease in $\beta$ and increase in $\gamma$ as well as slight increase in $\delta$, the deduced critical exponents are very close to the theoretical values of mean-field model, indicating the existence of a long-range interactions dominate the critical behavior around $T_{C}$.

Consequently, in mean-field model, we utilize the isothermal magnetic entropy change $\left(-\Delta S_{M}\right)$ in our system, obtained from isothermal magnetization measurements, to estimate the spontaneous magnetization of $\mathrm{Mn}_{0.96} \mathrm{Nb}_{0.04} \mathrm{CoGe}$. An excellent 
agreement is found between the spontaneous magnetization determined from the entropy change $\left(-\Delta S_{M}\right.$ vs. $\left.M^{2}\right)$ and the classical extrapolation from the Arrott curves $\left(H / M\right.$ vs. $\left.M^{2}\right)$, thus confirming that the magnetic entropy change is a valid approach to estimate the spontaneous magnetization in this system.

\section{EXPERIMENTAL DETAILS}

An ingot of polycrystalline $\mathrm{Mn}_{0.96} \mathrm{Nb}_{0.04} \mathrm{CoGe}$ was prepared by arc melting the appropriate amounts of $\mathrm{Mn}$ (99.9\%), Nb (99.999\%) powder, Co (99.9\%), and $\mathrm{Ge}$ $(99.999 \%)$ chips in an argon atmosphere. During arc melting, a 5\% excess Mn over the stoichiometric amount was added to compensate the weight loss of Mn. The polycrystalline ingot was melted several times to achieve good homogeneity. The ingot was then wrapped in tantalum foil, sealed in a quartz ampoule, and subsequently annealed at $850{ }^{\circ} \mathrm{C}$ for $120 \mathrm{~h}$ and then quenched in water at room temperature. The magnetization measurements were carried out using the vibration sample magnetometer option of a Quantum Design 14 T Physical Property Measurement System.

\section{RESULT AND DISCUSSION}

The temperature dependence [ $200 \mathrm{~K}$ to $340 \mathrm{~K}$ ] of the magnetization $M(T)$ of the $\mathrm{Mn}_{0.96} \mathrm{Nb}_{0.04} \mathrm{CoGe}$ alloy measured in a magnetic field of $100 \mathrm{Oe}$ is shown in Figure 1(a). All these data were taken in the warming run after zero-field cooling (ZFC), followed by field cooling (FC). The $M(T)$ curve exhibits a sharp FM-PM phase transition. The Curie temperature $\left(T_{C}\right)$, defined by the minimum in $d M / d T$, has been determined to be $T_{C}=269 \mathrm{~K}$ (the inset of Figure 1(a)). The magnetic entropy change, $-\Delta S_{M}$, of materials with second order transitions can be calculated reliably using the Maxwell relation. ${ }^{14}$ Figure $1(\mathrm{~b})$ shows the variation of $-\Delta S_{M}$ with temperature for the $\mathrm{Mn}_{0.96} \mathrm{Nb}_{0.04} \mathrm{CoGe}$ alloy. The maxima in the $-\Delta S_{M}$ versus $T$ curves are found to be in the vicinity of $\mathrm{T}_{\mathrm{c}}$ and is about $-2.95 \mathrm{~J} \mathrm{~kg}^{-1} \mathrm{~K}^{-1}$ for a magnetic field change of $5 \mathrm{~T}$, which is a bit less than that found for the undoped MnCoGe $\left(-4.8 \mathrm{~J} \mathrm{~kg}^{-1} \mathrm{~K}^{-1}\right.$ at $\left.5 \mathrm{~T}\right){ }^{9}$ Figure $1(\mathrm{c})$ shows the dependence of the magnetic entropy change on the parameter $\left(H / T_{C}\right)^{2 / 3}$. Mean-field theory predicts that $-\Delta S_{M}$ is proportional to $\left(H / T_{C}\right)^{2 / 3}$ in the vicinity of a second-order phase transition. ${ }^{15}$ The linear fit to the data in Figure 1(c) clearly demonstrates that the relationship $\Delta S_{M} \propto\left(H / T_{C}\right)^{2 / 3}$ is valid in the temperature region near $\mathrm{T}_{\mathrm{C}}$ for that system.

In order to further clarify the nature of the FM-PM phase transition, we performed an analysis of the critical behaviour near $T_{C}$. We measured the isothermal magnetization versus applied field around the Curie temperature, as shown in Figure 2(a). A plot of $H / M$ versus $M^{2}$, known as the standard Arrott plot, is shown in Figure 2(b) for the temperatures in the vicinity of $T_{C}$. According to the criterion proposed by Banerjee, ${ }^{16}$ the order of magnetic transition can be determined from the slope of the isotherm plot. For the $\mathrm{Mn}_{0.96} \mathrm{Nb}_{0.04} \mathrm{CoGe}$ alloy, the positive slope of the $H / M$ versus $M^{2}$ curves throughout the transition temperature indicates that the phase transition is second order. However, a close inspection of the Arrott plot reveals that not all of the curves
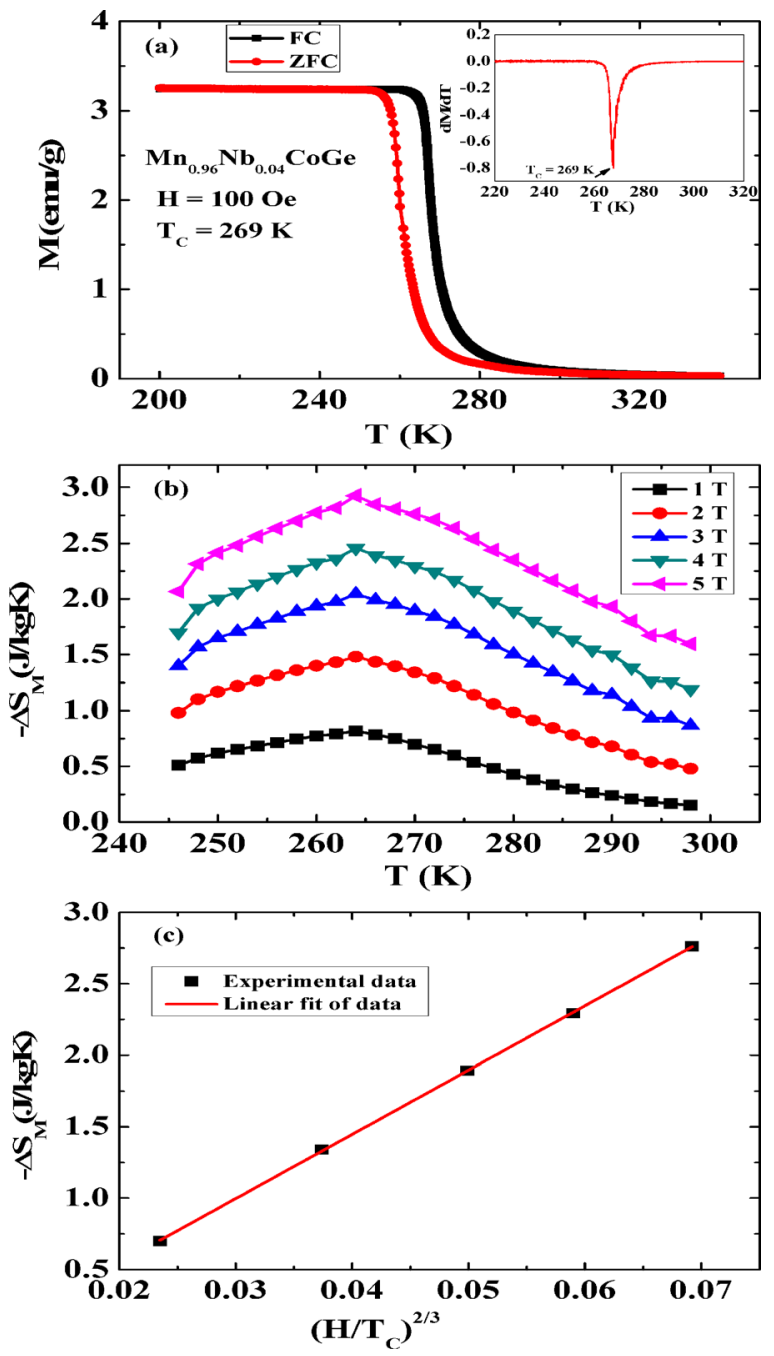

FIG. 1. (a) Temperature dependence of magnetization. The inset shows $d M / d T$ versus $T$ used for the determination of $T_{C}$. (b) Temperature dependence of the isothermal magnetic entropy change $-\Delta S_{M}(T, H)$ for $\mathrm{Mn}_{0.96} \mathrm{Nb}_{0.04} \mathrm{CoGe}$ calculated from magnetization isotherms. (c) Dependence of the magnetic entropy change on the parameter $\left(H / T_{C}\right)^{2 / 3}$. The solid line represents a linear fit to the data.

are parallel to each other, indicating that the critical exponents of $\beta=0.5$ and $\gamma=1$ are not satisfied. The scaling hypothesis postulates that a second-order magnetic phase transition near $T_{C}$ is characterized by a set of critical exponents, namely $\beta, \gamma$, and $\delta .{ }^{17}$ In this work, we have used different methods to investigate the critical behaviour of the $\mathrm{Mn}_{0.96} \mathrm{Nb}_{0.04} \mathrm{CoGe}$ alloy, namely, the modified Arrott plots (MAP) method, the Kouvel-Fisher (KF) method, critical isotherm analysis, and the Widom scaling relation method. The first method used to calculate the critical exponents is the MAP method, which is based on the Arrott-Noakes equation of state. ${ }^{18}$ Quantitative fits are made to the Arrott plots using the following equations: ${ }^{19}$

$$
\begin{gathered}
M_{S}(T)=\lim _{H \rightarrow 0}(M)=M_{0}(-\varepsilon)^{\beta}, \quad \varepsilon<0, \\
\chi_{0}^{-1}(T)=\lim _{H \rightarrow 0}(H / M)=\left(h_{0} / M_{0}\right) \varepsilon^{\gamma}, \quad \varepsilon>0,
\end{gathered}
$$

where $M_{0}$ and $h_{0}$ are constants and $\varepsilon=\left(T-T_{C}\right) / T_{C}$ is the reduced temperature. Initial values of $\beta$ and $\gamma$ are selected, 

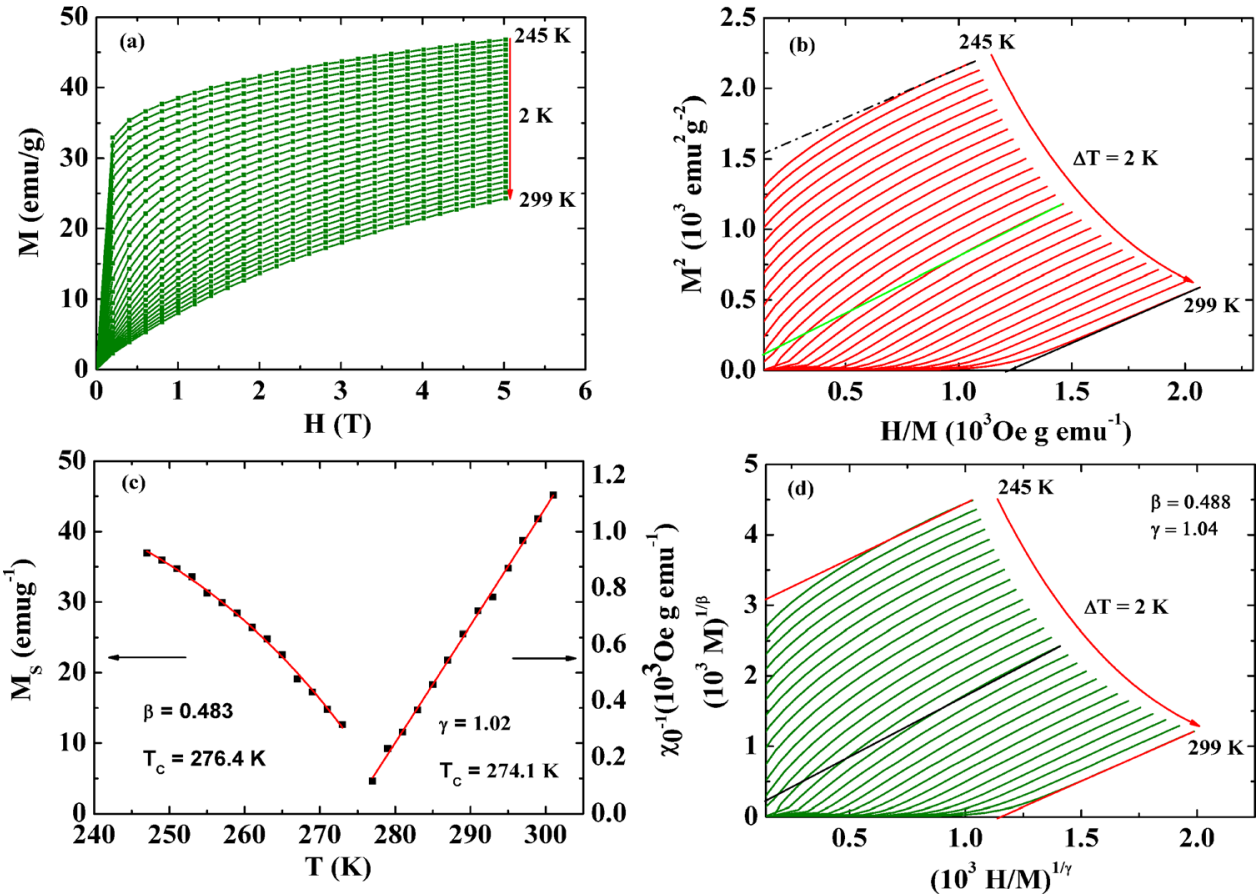

FIG. 2. (a) Isothermal magnetization curves for $\mathrm{Mn}_{0.96} \mathrm{Nb}_{0.04} \mathrm{CoGe}$ in the vicinity of $T_{C}$. (b) Arrott plot of $M^{2}$ vs $H / M$ at temperatures in the vicinity of $T_{C}$. (c) Temperature dependence of the spontaneous magnetization $M_{S}$ and inverse initial susceptibility $\chi_{0}^{-1}$ (solid lines are fitted to Eqs. (1) and (2). (d) Modified Arrott plot obtained by the Kouvel-Fisher method showing isotherms of $M^{1 / \beta}$ vs $\left(\frac{H}{M}\right)^{1 / \gamma}$ with the calculated $\beta=0.483$ and $\gamma=1.02$.

then a plot of $M^{1 / \beta}$ versus $(H / M)^{1 / \gamma}$ is obtained. $M_{S}$ is then determined from the intersection of the linearly extrapolated curve with the $M^{1 / \beta}$ axis.

It is imperative to note that only the high field linear region is used for the analysis because MAPs tend to deviate from linearity at low field due to the mutually misaligned magnetic domains. ${ }^{20} \mathrm{Next}, M_{S}$ is plotted as a function of temperature. To determine $\chi_{0}^{-1}(T)$, a similar procedure is used in conjunction with the $(H / M)^{1 / \gamma}$ axis. The values of $\chi_{0}{ }^{-1}(T)$ and $M_{S}(T)$ are plotted as a function of temperature as shown in Figure 2(c) and these plots are then fitted with Eqs. (1) and (2), thus obtaining values of $\beta$ and $\gamma$. These new critical exponent values are then used to construct new MAPs. These steps are repeated until the iterations converge to the optimum $\beta, \gamma$, and $T_{C}$ values. Using Eqs. (1) and (2), the MAPs shown in Figure 2(c) yielded critical parameters, $\beta=0.483 \pm 0.02$ and $\gamma=1.02 \pm 0.04$. The modified Arrott plots are drawn in Figure 2(d), which shows clearly that all lines are parallel to each other.

The Kouvel-Fisher method, which makes use of Eqs. (3) and (4) shown below, is a more accurate way of determining the critical exponents $\beta$ and $\gamma^{21}$

$$
\begin{gathered}
\frac{M_{S}(T)}{d M_{S}(T) / d T}=\frac{T-T_{C}}{\beta}, \\
\frac{\chi_{0}^{-1}(T)}{d \chi_{0}^{-1}(T) d T}=\frac{T-T_{C}}{\gamma} .
\end{gathered}
$$

According to the above equations, plotting $M_{S}(T)\left[d M_{S} / d T\right]^{-1}$ and $\chi_{0}^{-1}(T)\left[d \chi_{0}^{-1} / d T\right]^{-1}$ versus temperature yields straight lines with slopes of $1 / \beta$ and $1 / \gamma$, respectively, as shown in Figure 3(a). The critical exponents $\beta$ and $\gamma$, and $T_{C}$ obtained using the Kouvel-Fisher method, are $\beta=0.488, T_{C}=275.1 \mathrm{~K}$ and $\gamma=1.04, T_{C}=274.4 \mathrm{~K}$. A comparison of the critical exponents $\beta$ and $\gamma$ obtained using the MAPs and those obtained using the KF method reveals that these values match reasonably well. The value of the critical component $\delta$ can be determined directly from the critical isotherm $M\left(T_{C}, H\right)$ according to the below equation

$$
M_{T_{C}}=D H^{1 / \delta}, \quad \varepsilon=0, T=T_{C} .
$$

Figure 3(b) shows the magnetic field dependence of magnetization at $T_{C}=275 \mathrm{~K}$ for the $\mathrm{Mn}_{0.96} \mathrm{Nb}_{0.04} \mathrm{CoGe}$ alloy. The inset shows the critical isotherm on a $\log -\log$ scale. From Eq. (5), a plot of $\log (M)$ versus $\log (H)$ is expected be a straight line with slope $1 / \delta$. The obtained $\delta$ value from the critical isotherm is 3.125 . Another way of obtaining the critical component $\delta$ is by using the Widom scaling relation shown in the following equation:

$$
\partial=1+\frac{\gamma}{\beta} .
$$

Using Eq. (5) and the critical parameters $\beta$ and $\gamma$ obtained using the MAPs and those obtained using the KF method, the deduced $\delta$ values are 3.11 and 3.13, respectively. Thus, the Widom scaling relation has confirmed the reliability of the critical exponents deduced from the experimental data. The reliability of the calculated exponents $\beta$ and $\gamma$ can be confirmed by using the scaling theory. In the critical region, according to the scaling theory, the magnetic equation of state can be written as

$$
M(H, \varepsilon)=\varepsilon^{\beta} f_{ \pm}\left(H / \varepsilon^{\beta+\gamma}\right),
$$



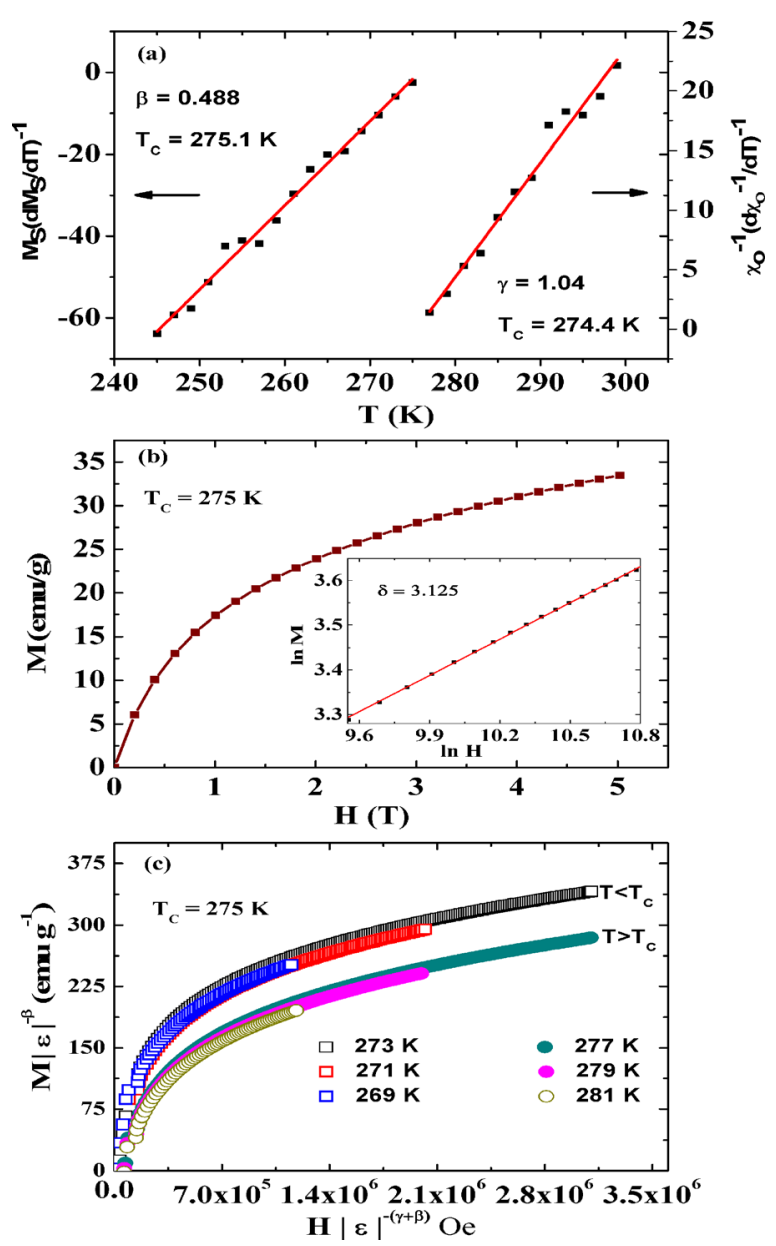

FIG. 3. (a) Kouvel-Fisher plot for the spontaneous magnetization $M_{S}(T)$ and the inverse initial susceptibility $\chi_{0}^{-1}(\mathrm{~T})$ (solid lines are fitted to Eqs. (3) and (4)). (b) The critical isotherm analysis at $T_{C}$. The inset shows the same plots on the log-log scale (solid line is a linear fit to Eq. (5). (c) Scaling plots indicating universal curves below and above $T_{C}$ for the $\mathrm{Mn}_{0.96} \mathrm{Nd}_{0.04} \mathrm{CoGe}$ alloy.

where $\varepsilon$ is the reduced temperature $\left(T-T_{C}\right) / T_{C}$ and $f_{+}$and $\mathrm{f}_{-}$ are regular analytical functions above and below $T_{C}$, respectively. Using $\beta$ and $\gamma$ obtained from the Kouvel-Fisher method, the plots of $M / \varepsilon^{\beta}$ versus $H / \varepsilon^{(\beta+\gamma)}$ as shown in Figure 3 (c) yield two universal curves, one for temperatures above $T_{C}$ and the other one for temperatures below $T_{C}$, in agreement with the scaling theory. This, therefore, confirms that the obtained values of the critical components as well as the $T_{C}$ value are reliable and in agreement with the scaling hypothesis.

The mean field interaction model for long range ordering has theoretical critical exponents of $\beta=0.5, \gamma=1.0$, and $\delta=3.0{ }^{7}$ The $\delta, \beta, \gamma$ values derived for the $\mathrm{Mn}_{0.96} \mathrm{Nd}_{0.04} \mathrm{CoGe}$ alloy are close to the mean field values, thus indicating that long range interactions dominate the critical behavior around $T_{C}$ for this compound. Thus, the critical behaviour analysis in the vicinity of $T_{C}$ determined that the magnetism of the $\mathrm{Mn}_{0.96} \mathrm{Nb}_{0.04} \mathrm{CoGe}$ alloy is governed by long range interactions, which is in agreement with the linear fit to the data in Figure 1(c), which clearly demonstrates that the relationship $\Delta S_{M} \propto\left(H / T_{C}\right)^{2 / 3}$ is valid around $T_{C}$. It is also speculated that the competition between the localized Mn-Mn magnetic interactions should be responsible for the critical behavior in this system.
A general result issued from a mean-field theory reveals that the dependence of the magnetic entropy on the relative magnetization can be described as $^{22,23}$

$$
\begin{aligned}
S(\sigma)= & -N k_{B}\left[\ln (2 J+1)-\ln \left(\frac{\sinh \left(\frac{2 \mathrm{~J}+1}{2 \mathrm{~J}}\right) \mathrm{B}_{\mathrm{J}}^{-1}(\sigma)}{\sinh \left(\frac{1}{2 J} \mathrm{~B}_{\mathrm{J}}^{-1}(\sigma)\right)}\right)\right. \\
& \left.+\mathrm{B}_{\mathrm{J}}^{-1}(\sigma) \cdot \sigma\right],
\end{aligned}
$$

where $N$ is the number of spins, $J$ the spin values, $k_{B}$ the Boltzmann constant, $\sigma$ the relative magnetization ${ }^{24}(\sigma=M /$ $\left.\left(g \mu_{B} J N\right)\right)$, and $B_{J}$ the Brillouin function for a given $J$ value.

From a power expansion of Eq. (8), $\Delta S_{M}$ is proportional to $M^{2}$ and, from the mean-field model, for small $M$ values $^{22,25}$

$$
-\mathrm{S}(\sigma)=\frac{3 \mathrm{~J}}{2 \mathrm{~J}+1} \mathrm{Nk}_{\mathrm{B}} \sigma^{2}+\mathrm{O}\left(\sigma^{4}\right) .
$$

Furthermore, the compound has a spontaneous magnetization below $T_{C}$ (ferromagnetic state) and consequently the $\sigma=0$ state is never attained. Explicitly, and considering only the first term of the expansion of Eq. (9), this corresponds to

$$
-\mathrm{S}(\sigma)=\frac{3 \mathrm{~J}}{2 \mathrm{~J}+1} N k_{B}\left(\sigma^{2}-\sigma_{\text {spon }}^{2}\right),
$$

which results in a shift of the isothermal $\Delta S_{M}$ vs. $M^{2}$ plots in the ferromagnetic region, with a horizontal drift from the origin corresponding to the value of $M^{2}$ spont. (T), while for $T>T_{c}$ the $\Delta S_{M}$ vs. $M^{2}$ plots start at a null $M$ value.

On the other hand, from bulk isothermal magnetization measurements $\Delta S_{M}$ values are calculated directly from the use of the well known Maxwell relation

$$
\Delta S_{M}(T, H)=S(T, H)-S(T, 0)=\int_{0}^{H}\left(\frac{\partial \mathrm{M}}{\partial \mathrm{T}}\right)_{\mathrm{H}} d H .
$$

If the $\left(-\Delta S_{M}\right)$ vs. $M^{2}$ plots show a linear dependence with constant slope throughout the experimental temperature/field range, this corresponds to the validity of the linear expansion of Eq. (10) or, in Landau theory, Eq. (11).

Values of the isothermal magnetic entropy change $\left(-\Delta S_{M}\right)$, taken from isothermal magnetization measurements, are used to estimate the spontaneous magnetization of $\mathrm{Mn}_{0.96} \mathrm{Nb}_{0.04} \mathrm{CoGe}$ using mean-field theory of entropy change. As discussed above, we have the basis to determine the spontaneous magnetization using the linear fits of the isothermal $\left(-\Delta S_{M}\right)$ vs. $M^{2}$ plots inside the ferromagnetic region. From magnetization and $\left(-\Delta S_{M}\right)$ data, plots of $\left(-\Delta S_{M}\right)$ vs. $M^{2}$ can be drawn as shown in Figure 4(a), showing a linear dependence with an almost constant slope throughout the ferromagnetic region. This magnitude of the slope is approximately 26.1, corresponding to a Curie constant of $0.0301 \mathrm{emuKOe}^{-1} \mathrm{~g}^{-1}$. The spontaneous 

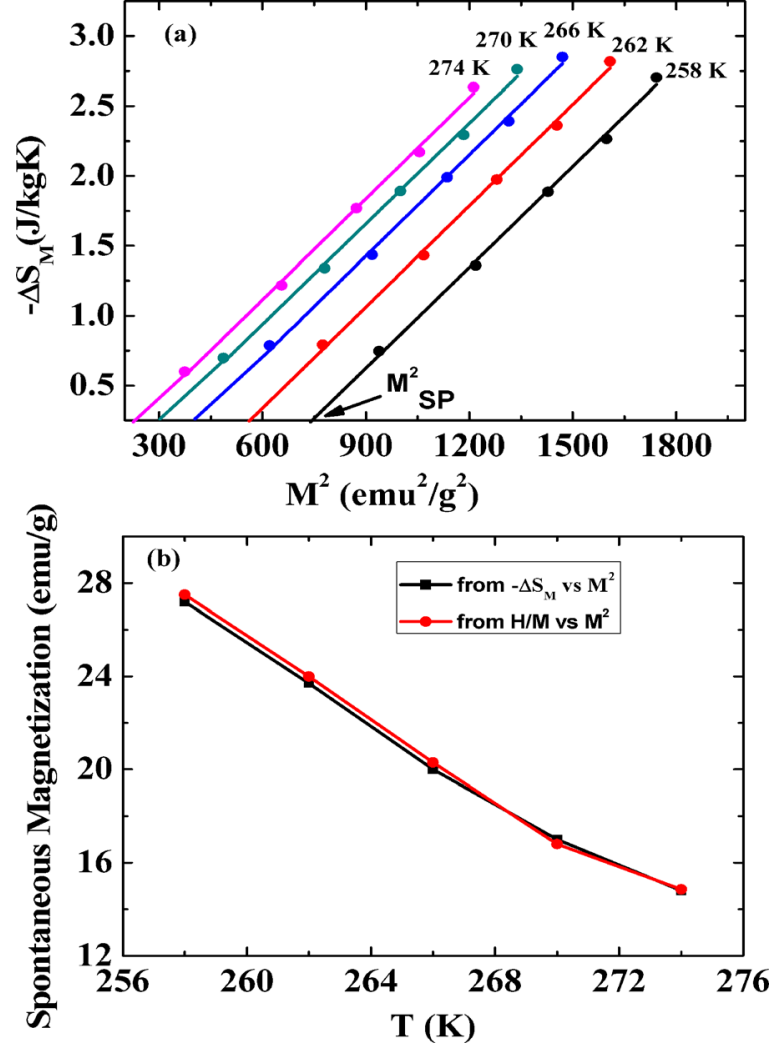

FIG. 4. (a) Isothermal $-\Delta S_{M}$ vs. $M^{2}$ curves for $\mathrm{Mn}_{0.96} \mathrm{Nb}_{0.04} \mathrm{CoGe}$. Solid lines are linear fits to data. (b) Spontaneous magnetization of $\mathrm{Mn}_{0.96} \mathrm{Nb}_{0.04} \mathrm{CoGe}$, estimated from the extrapolation of the $\left(-\Delta S_{M}\right.$ vs. $\left.M^{2}\right)$ curves and from the Arrott plots $\left(H / M\right.$ vs. $\left.M^{2}\right)$.

magnetization $M_{S}(T)$ is then estimated, and compared to the results obtained from the Arrott curves, as shown in Figure 4(b). The excellent agreement between the two methods confirms the validity of this process to estimate the spontaneous magnetization using a mean-field analysis of the magnetic entropy change in $\mathrm{Mn}_{0.96} \mathrm{Nb}_{0.04} \mathrm{CoGe}$ system.

\section{CONCLUSIONS}

In summary, the critical phenomena of $\mathrm{Mn}_{0.96} \mathrm{Nb}_{0.04} \mathrm{CoGe}$ alloy have been comprehensively studied. The values deduced for the critical exponents are close to the theoretical prediction of the mean-field model values, thus indicating that long range interactions dominate the critical behavior in the system. The validity of the calculated critical exponents was also confirmed by the scaling theory. The methodology based on the analysis of the magnetic entropy change $\left(-\Delta S_{M}\right.$ vs. $M^{2}$ ), compared with the classical extrapolation of the Arrott curves $\left(H / M\right.$ vs. $\left.M^{2}\right)$, confirms that the magnetic entropy change is a valid method to determine the spontaneous magnetization of the $\mathrm{Mn}_{0.96} \mathrm{Nb}_{0.04} \mathrm{CoGe}$ system and in other compounds as well.

\section{ACKNOWLEDGMENTS}

J.C.D. acknowledges the NRF (Grant No: 85115) for an Innovation Postdoctoral Fellowship, supervised by A.M.S.

${ }^{1}$ M. H. Phan and S. C. Yu, J. Magn. Magn. Mater. 308, 325 (2007).

${ }^{2}$ A. Kitanovski and P. W. Egolf, J. Magn. Magn. Mater. 321, 777 (2009).

${ }^{3}$ H. Wada and Y. Tanabe, Appl. Phys. Lett. 79, 3302 (2001).

${ }^{4}$ M. Balli, D. Fruchart, D. Gignoux, and R. Zach, Appl. Phys. Lett. 95, 072509 (2009).

${ }^{5}$ V. S. Amaral and J. S. Amaral, J. Magn. Magn. Mater. 272-276, 2104 (2004).

${ }^{6}$ S. Kallel, N. Kallel, O. Peña, and M. Oumezzine, Mater. Lett. 64, 1045 (2010).

${ }^{7}$ V. Franco, A. Conde, E. J. M. Romero, and J. S. Blazquez, J. Phys.: Condens. Matter 20, 285207 (2008).

${ }^{8}$ Q. Y. Dong, H. W. Zhang, J. R. Sun, B. G. Shen, and V. Franco, J. Appl. Phys. 103, 116101 (2008).

${ }^{9}$ N. T. Trung, L. Zhang, L. Caron, K. H. Buschow, and E. Bruck, Appl. Phys. Lett. 96, 172504 (2010).

${ }^{10}$ C. L. Zhang, D. H. Wang, Q. Q. Cao, Z. D. Han, H. C. Xuan, and Y. W. Du, Appl. Phys. Lett. 93, 122505 (2008).

${ }^{11}$ T. Kanomata, H. Ishigaki, T. Suzuki, H. Yoshida, S. Abe, and T. Kaneko, J. Magn. Magn. Mater. 140, 131 (1995).

${ }^{12}$ J. Y. Fan, L. S. Ling, B. Hong, L. Zhang, L. Pi, and Y. H. Zhang, Phys. Rev. B 81, 144426 (2010).

${ }^{13}$ R. Cabassi, F. Bolzoni, A. Gauzzi, and F. Licci, Phys. Rev. B 74, 184425 (2006).

${ }^{14}$ N. A. De Oliveira and P. J. Von Ranke, Phys. Rev. B 77(21), 214439 (2008).

${ }^{15}$ J. L. Wang, S. J. Campbell, R. Zeng, C. K. Poh, and S. X. Dou, J. Appl. Phys. 105, 07A909 (2009).

${ }^{16}$ S. K. Banerjee, Phys. Lett. 12, 16 (1964).

${ }^{17}$ H. E. Stanley, Introduction to Phase Transitions and Critical Phenomena (Oxford University Press, London, 1971).

${ }^{18}$ A. Arrott and J. E. Noakes, Phys. Rev. Lett. 19, 786 (1967).

${ }^{19}$ M. Sahana, U. K. Rossler, N. Ghosh, S. Elizabeth, H. L. Bhat, K. Dorr, D. Eckert, M. Wolf, and K. Muller, Phys. Rev. B 68, 144408 (2003).

${ }^{20} \mathrm{~A}$. Aharoni, Introduction to the Theory of Ferromagnetism (Oxford University Press, New York, 1971).

${ }^{21}$ J. S. Kouvel and M. E. Fisher, Phys. Rev. 136, A1626 (1964).

${ }^{22}$ J. S. Amaral, N. J. O. Silva, and V. S. Amaral, J. Magn. Magn. Mater. 322, 1569 (2010).

${ }^{23}$ A. M. Tishin and Y. I. Spichin, The Magnetocaloric Effect and Its Applications (IOP Publishing, London, 2003).

${ }^{24}$ G. F. Wang, L. Song, Z. Q. Ou, Z. R. Zhao, and O. Tegus, Acta Metall. Sin. (Engl. Lett.) 20, 265 (2007).

${ }^{25}$ H. Oesterreicher and F. T. Parker, J. Appl. Phys. 55, 4334 (1984). 